\title{
The Use of Ionization Electron Columns for Space-Charge Compensation in High Intensity Proton Accelerators
}

\author{
V.Shiltsev ${ }^{\mathrm{a}}$, Y.Alexahin ${ }^{\mathrm{a}}$, V.Kamerdzhiev ${ }^{\mathrm{a}}$, V.Kapin ${ }^{\mathrm{a}}$, G.Kuznetsov ${ }^{\mathrm{a}}$ \\ ${ }^{a}$ Fermilab, PO Box 500, Batavia, IL, 60510, USA
}

\begin{abstract}
We discuss a recent proposal to use strongly magnetized electron columns created by beam ionization of the residual gas for compensation of space charge forces of high intensity proton beams in synchrotrons and linacs. The electron columns formed by trapped ionization electrons in a longitudinal magnetic field that assures transverse distribution of electron space charge in the column is the same as in the proton beam. Electrostatic electrodes are used to control the accumulation and release of the electrons. Ions are not magnetized and drift away without affecting the compensation. Possible technical solution for the electron columns is presented. We also discuss the first numerical simulation results for space-charge compensation in the FNAL Booster and results of relevant beam studies in the Tevatron.
\end{abstract}

Keywords: space-charge effects, compensation, electron columns

PACS: 29.27.Bd, 41.75.Fr, 41.75.Ew

\section{INTRODUCTION: COMPENSATION OF SPACE CHARGE EFFECTS}

It is long known that compensation of space charge forces in high current proton beams is possible if the amount of electrons stored in the beam is equal to

$$
<\eta>=1 / \gamma^{2}
$$

where $\eta$ is relative fraction of the electron charge w.r.t. the proton charge, or degree of charge compensation; and $\gamma$ is relativistic factor of protons [1] . Conditions for effective space charge compensation (SCC) are that the electron charge distribution is the same as the proton one (preferably in all three dimensions) and that the system of electrons and protons is dynamically stable. Electron lenses were suggested as a possible instrument for such compensation [2] but the method is technologically challenging and has certain limits, particularly, it requires a large number of expensive lenses for high periodicity machines. Partial neutralization by ionization electrons those which are born by ionization of residual gas by charged beam - was attempted before with some success but the stability criteria was not easily assured [3]. The reason is that a system consisting of a proton beam and free electrons becomes unstable at high proton intensities due to so called "electron cloud" effects which include the increase of vacuum pressure, emittance growth and proton beam instability. 
This difficulty can be overcome if protons and electrons are immersed in the longitudinal magnetic field which is a) strong enough to keep electrons from escaping from the transverse position they are born at; b) strong enough to suppress the e-p instability; d) weak enough to allow ions escape and not affect the process of charge compensation. One possibility to arrange that is to have toroidal solenoid accelerator, e.g. a modified betatron [4], where longitudinal magnetic field provides beam focusing all along the beam orbit. Unfortunately, such machines are not widely accepted as practical as the acceleration of particles in them should cope with significant difficulties due to multiple resonance crossings. For most common existing accelerators where high energy protons are guided and focused by transverse fields of magnetic dipoles and quadrupoles, there is a possibility to satisfy condition (1) on average by having many space charge (SC) compensating elements around the ring ideally, one per every superperiod of the focusing lattice. In that case, the fraction of the ring circumference $C$ occupied by electron columns should be equal to

$$
R=1 /\left(\gamma^{2} \eta_{0}\right)
$$

where $\eta_{0}$ is the degree of local SC compensation, e.g. if $\eta_{0}=1-$ full charge compensation inside solenoids - then, $R=1 / \gamma^{2}$.

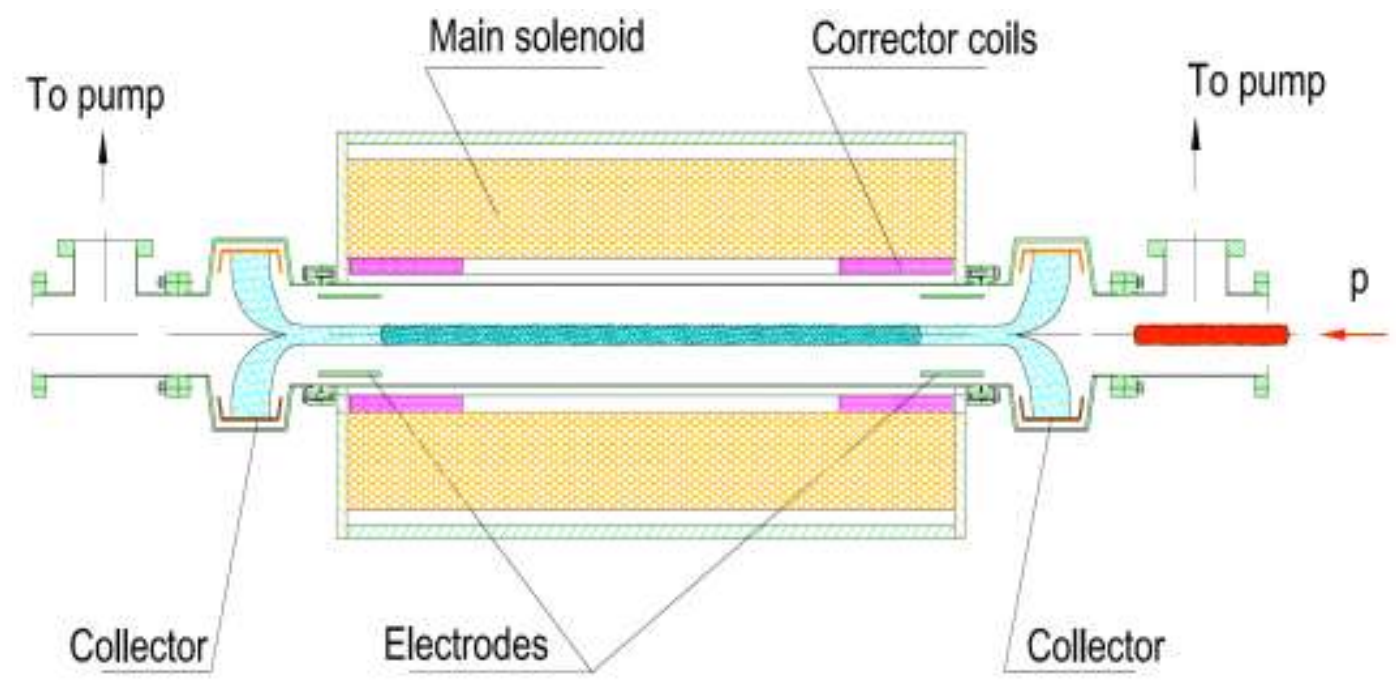

FIGURE 1. A possible layout of electron column for space charge compensation.

Schematically, the compensation device - an "electron column" [5] - may look as shown in Fig.1 and consist of a solenoid magnet, a pair of ring- or cylinder-shape electrodes to control the accumulation of electrons, a controlled leak to vary vacuum conditions at the location of the electron column (EC), electron collectors and vacuum ports for possible differential pumping. In the most straightforward configuration, the variation of magnetic field and electrostatic potential along the e-column may look as depicted in Fig.2 (left). If the B-field is strong enough, than transverse charge distribution of accumulated ionization electrons follows the proton one as shown in Fig.2 (right) for $\eta_{0}=0.5,1,1.5$. The system of electrodes may be more complex than just two rings and, for example provide desired distribution of potential along the $\mathrm{z}$ axis. Voltages can be made time-dependent to track changes of proton beam 
parameters (energy, size, etc). The solenoid field distribution can be varied too in order to reduce $\mathrm{x}-\mathrm{y}$ coupling it introduces.
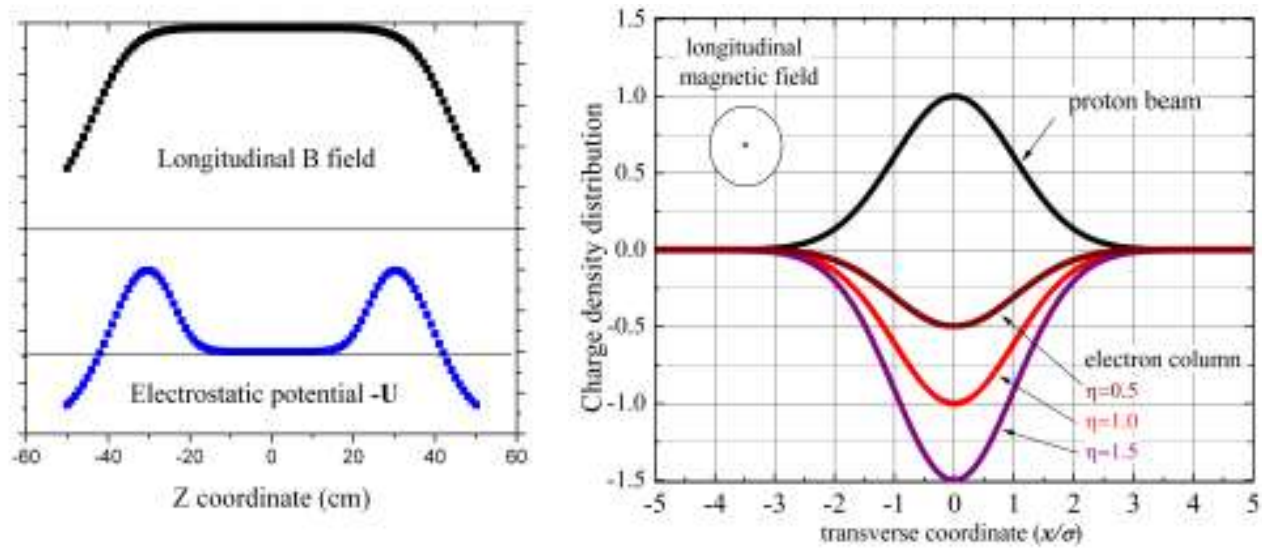

FIGURE 2. Longitudinal variations of magnetic field and electrostatic potential in the electron column (left). Transverse distribution of charge in a circulating proton beam and in a strongly magnetized column of ionization electrons with various degrees of local charge compensation (right)

\section{PARAMETERS OF ELECTRON COLUMNS}

To achieve the degree of the SCC equal to $f$ in a ring with $N_{e c}$ electron columns, the total accumulated charge in each column should be:

$$
Q_{\text {e.c. }}=-\frac{|e| h N_{b} B_{f}}{N_{e c}\left(\gamma^{2}-1\right)} f
$$

where $h$ is the RF harmonic number and $N_{b}$ is number of protons/bunch, and $B_{f}$ is the bunching factor defined as a ratio of peak to average proton current within one RF bucket. If the length of one column is $L_{e c}$ then the electrostatic barrier voltage of - $U$ will result in accumulation of the total electron charge of:

$$
Q_{e . c .}[C]=\frac{U[V]}{30\left(1+2 \ln \frac{b}{a}\right)} \cdot \frac{L_{e c}}{c}
$$

where $b$ and $a$ are the radii of the vaccum beam pipe and the electron beam, correspondingly. Comparing Eqs.(3) and (4) one gets the voltage required for the SCC which is proportional to peak proton bunch current $J_{p}{ }^{\text {peak }}$ :

$$
U[V]=f \cdot 30 J_{p}^{\text {peak }}[A] \cdot\left(1+2 \ln \frac{b}{a}\right) \frac{C \gamma}{L_{e c} N_{e c}\left(\gamma^{2}-1\right)^{3 / 2}}
$$

Table 1 below presents possible electron column parameters needed for full SCC in three different proton synchrotrons (it is assumed that $1+2 \ln (\mathrm{b} / \mathrm{a})$ is about $5, P_{s}$ is the focusing lattice super-period).

TABLE 1. Electron columns for various acce

\begin{tabular}{lccccccc}
\hline Accelerator & Kin.Energy & $\boldsymbol{C}$ & $\boldsymbol{P}_{\boldsymbol{s}}$ & $\boldsymbol{J}_{\boldsymbol{p}} \boldsymbol{p e a k}^{\boldsymbol{e}}$ & $\boldsymbol{N}_{\boldsymbol{e c}}$ & Length $\boldsymbol{L}_{\boldsymbol{e c}}$ & $\boldsymbol{-} \boldsymbol{U}[\boldsymbol{k} \boldsymbol{V}]$ \\
\hline FNAL Booster & $400 \mathrm{MeV}$ & $474 \mathrm{~m}$ & 24 & $1 \mathrm{~A}$ & 24 & $1 \mathrm{~m}$ & 4.0 \\
CERN PS & $1.4 \mathrm{GeV}$ & $628 \mathrm{~m}$ & 10 & $4 \mathrm{~A}$ & 10 & $1 \mathrm{~m}$ & 7.9 \\
FNAL MI\&RR & $8.0 \mathrm{GeV}$ & $3.3 \mathrm{~km}$ & 1 & $3 \mathrm{~A}$ & 4 & $1 \mathrm{~m}$ & 4.7 \\
\hline
\end{tabular}


The time needed to generate enough electrons to compensate the proton space charge - so called "neutralization time" - is equal to $\tau=0.05$ [s]/P[nTorr] for $\mathrm{H} 2$ dominated vacuum. For the purpose of the SCC on average (without the goal of tracking the bunch current profile), the accumulation time $\tau$ should be of the order of $0.1 \mathrm{~ms}$ (characteristic time scale of beam parameters variation due to acceleration) that calls for the local vacuum pressure of in $\mathrm{EC}$ of about $\mathrm{P}=5 \mathrm{e}-7$ Torr.

It is important that Larmor radius of ionization electrons $r_{L}=p c / e B$ is smaller than the proton beam radius (so that the electron distribution was not smeared off the proton distribution). Characteristic energy of the ionization electrons is about $-e U$, so the Larmor radius of $8 \mathrm{keV}$ electrons in $B=30 \mathrm{kG}$ is about $0.1 \mathrm{~mm}$ - that is much less than beam radii in the accelerators of interest (few $\mathrm{mm} \mathrm{rms}$ ).

Coupling (in the units of tune-split) introduced by one solenoid is about $\kappa=$ $(B L) /(4 \pi B \rho)$ that is about 0.15 for $30 \mathrm{kG} 1 \mathrm{~m}$ long solenoid in $1 \mathrm{GeV}$ machine - and it can be compensated either by skew correctors or - even easier - by proper choice of $B$-field directions in different ECs.

The electron column will slowly rotate around its axis under impact of its own space charge electric field $E_{s c}$ and external magnetic field $B$ with drift velocity :

$$
v_{d}=c \frac{E_{s c}}{B}
$$

which is about $0.03 \%$ of $c$ for the beams we considered above and $B=30 \mathrm{kG}$. Because of such rotation it seems to be beneficial to install ECs at the locations where proton beams have about equal horizontal and vertical dimensions.

Besides promise of better $e-p$ stability, high magnetic field leads to fast cooling of electrons. Indeed, the power of synchrotron radiation is:

$$
W=\frac{4 e^{4} B^{2}}{3 m^{3} c^{5}} \cdot \frac{m V_{\perp}^{2}}{2}=\frac{E_{k i n}}{\tau_{S R}}
$$

and the corresponding cooling time at, e.g., $\mathrm{B}=30 \mathrm{kG}$ is about $0.3 \mathrm{~s}$.

\section{MODELING OF SCC WITH ELECTRON COLUMNS}

Modeling of the SCC with electron columns in Fermilab's Booster synchrotron has been performed using Mathematica and MAD8 codes [6]. The "electron columns" were represented by equidistant thin beam-beam elements with equal charges. Their transverse sizes were assumed to coincide with the beam sizes at the particular location as determined by the local beta-functions and current values of emittances. The "columns" were placed inside defocusing magnets upstream long drifts where the beta-functions are approximately equal: $\beta_{x} \approx 13 \mathrm{~m}, \beta_{y} \approx 15 \mathrm{~m}$. Time dependence of the columns charge just repeated the central bunch density as it varies during bunching, to achieve space charge compensation factor $f$.

Fig.3 presents tracking results with bunch intensity $N_{b}=6 \mathrm{e} 10,24$ "electron columns" and total compensation factor $f$ equal to 0 or 0.5 . The plots in the left column show normalized beam intensity vs turn number and the plots in the right column show emittances normalized to the initial values (red - horizontal, blue vertical). 
With "electron columns" placed in each of the 24 Booster periods, the space charge compensation drastically reduces emittance blowup and beam losses. However, even in the case of $f=1$ there is still noticeable effect of space charge since localized columns provide compensation only on average [6]. With twice smaller number of "columns" (12), the beneficial effect of compensation is somewhat smaller for the same values of the compensation factor $f$.

It must be noted that the presented results are obtained by $2 \mathrm{D}$ simulations, the synchrotron motion was taken into account only as precalculated bunching factor. The space charge gradient modulation along the bunch may introduce synchro-betatron coupling and produce an adverse affect on particle stability.
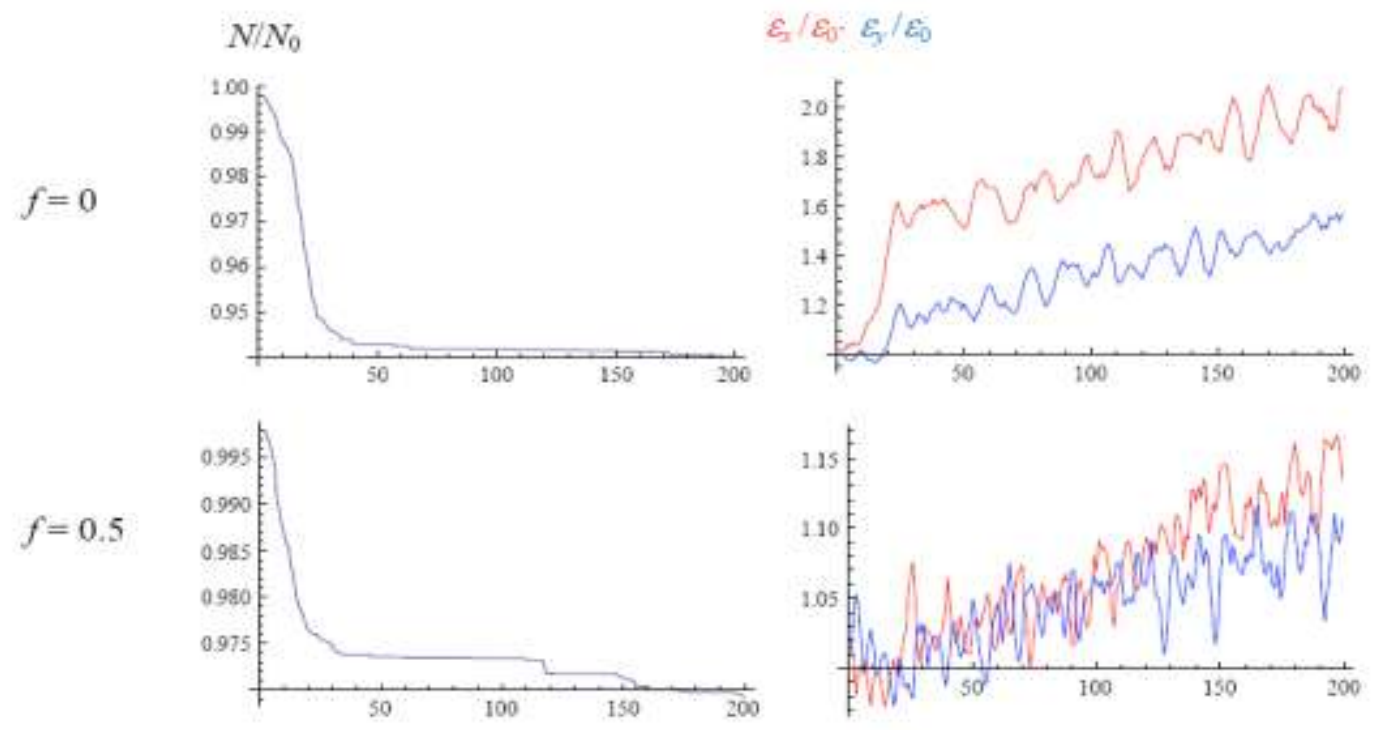

FIGURE 3. Fermilab Booster ring simulation over 200 turns without space-charge compensation (top) and with $50 \%$ compensation by 24 localized electron columns (from [5]). On the left - normalized intensity loss; on the right - growth of vertical and horizontal emittances normalized to their initial intensities. Booster bunch intensity $N_{b}=6 \cdot 10^{10}, n_{\text {columns }}=24$.

\section{FIRST TESTS OF ELECTRON COLUMNS IN THE TEVATRON}

Two Tevatron Electron Lenses (TELs) are installed in the Tevatron for the purpose of beam-beam compensation. They are described in great detail in $[7,8]$. The TELs employ strong solenoids and have electrostatic electrodes which can be used to arrange the configuration similar to what is depicted in Fig.1. The Tevatron injection energy is $150 \mathrm{GeV}$ and space charge effects for proton beams are completely negligible, but accumulation of ionization electrons inside the TEL solenoid should result in positive shift of betatron frequencies. Observation of such a tuneshift was the goal of a short study.

The Tevatron vacuum pressure is very good ( 2 nTorr in the TEL-1 location) and in order to get faster ionization electron accumulation rates we spoiled he vacuum by bombarding the vacuum pipe surfaces with the electron beam generated by the TEL electron gun. That allowed to increase the pressure up to 20 nTorr for short periods of time (minutes). During these periods, we monitored the Schottky spectra of the proton 
beam, see Fig.4, and observed the increase of the average tune of the beam by approximately 0.004 . That indicated accumulation of negative charge (electrons) in a significantly dense electrons column. The distance between containing $\mathrm{HV}$ electrodes is about $2 \mathrm{~m}$, the maximum voltage on them was $-2.6 \mathrm{kV}$.

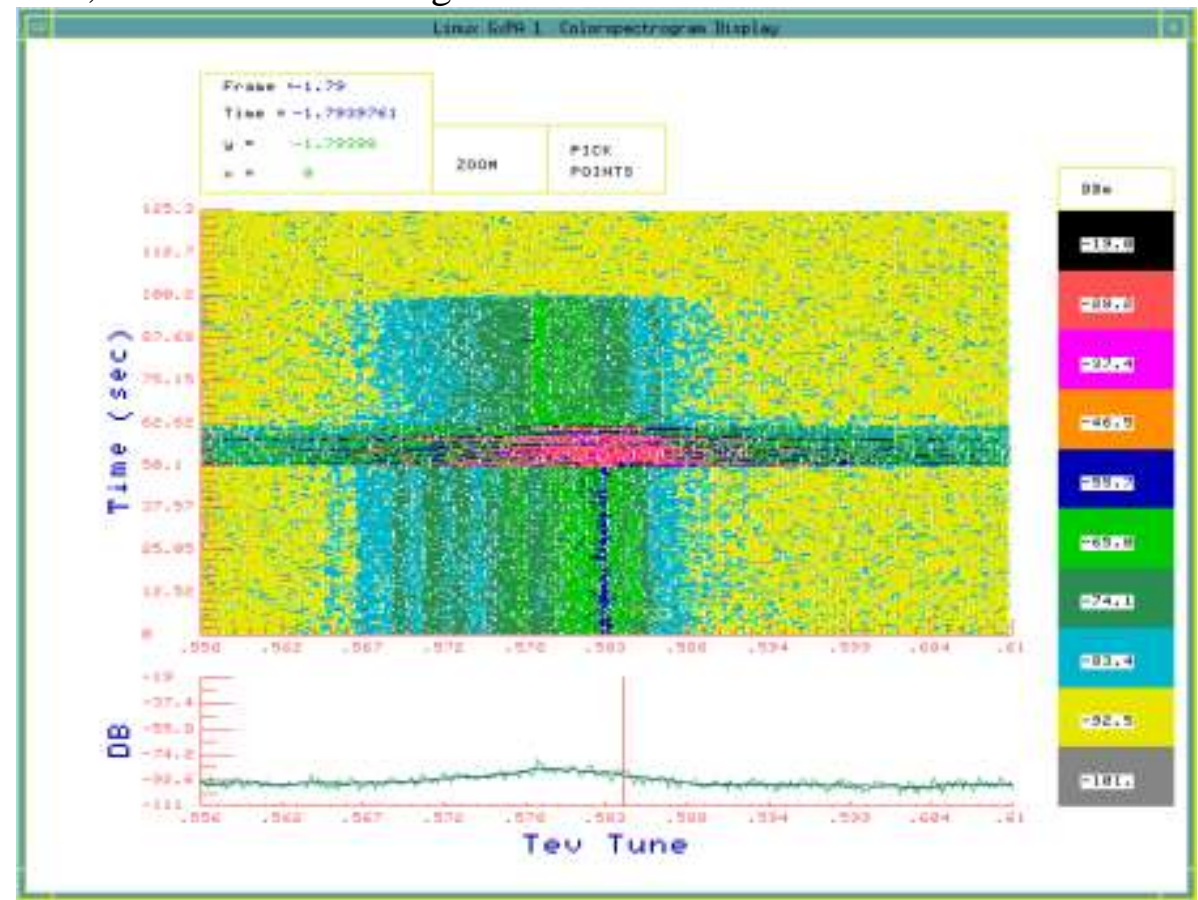

FIGURE 4. Color coded $21 \mathrm{MHz}$ Schottky spectra of the vertical motion of the $150 \mathrm{GeV}$ beam of protons in the Tevatron during space-charge neutralization study. The beam injection time is at the top ("waterfall" plot). Range of the vertical tunes on horizontal axis from 0.556 to 0.61 . Some $40 \mathrm{sec}$ after injection vacuum was worsened in the Tevatron Lectron Lens setup and the Schottky power went up. After that, the average tune of the beam increased by approximately 0.004 .

We plan to proceed both with theory and computer modeling of the space charge compensation by electron columns (particularly, explore electron dynamics and apply the method to FNAL Main Injector and/or Recycler) as well as continue experimental studies in the Tevatron with the TEL where a better way to increase vacuum pressure up to 100-1000 nTorr needs to be implemented. Decision on the prototyping and experimental test of SCC with ECs will be made upon successful results of these studies.

\section{REFERENCES}

1. G.Budker, Atomic Energy, 1, 5 (1956)

2. A.Burov, G.W.Foster, V.Shiltsev, Preprint Fermilab TM-2125 (2000)

3. V.Dudnikov, in ICFA-Hadron Beams 2002 Workshop, edited by M. D. Furnish et al., AIP Conference Proceedings 642, American Institute of Physics, Melville, NY, 2002, pp.367-369.

4. Yu.Korotaev. et al, in Proc. EPAC'1998, p.1061.

5. V.Shiltsev, in IEEE-Particle Accelerator Conference 2007, Albuquerque, 2007 pp.115901160.

6. Y.Alexahin, V.Kapin, "Study of Possibility of Space Charge Compensation in the Fermilab Booster with Multiple Electron Columns", beams-doc-3106 (2008), at http://beamsdoc.fnal.gov

7. V.Shiltsev et al., Phys. Rev. ST-AB 2, 071001 (1999)

8. V.Shiltsev, et al., Phys.Rev.Lett. 99, 244801 (2007), also in New Journ. of Phys.10, 043042 (2008) 